\title{
THE FIRST EXOTIC CLASS OF A MANIFOLD
}

\author{
BY \\ DAVID L. FRANK( $\left.{ }^{1}\right)$
}

Let $B F$ be the classifying space for stable oriented spherical fibrations. Gitler and Stasheff have defined a cohomology class $e_{1}$ in $H^{p r-1}\left(B F ; Z_{p}\right)$, where here (and throughout this paper) $p$ is an odd prime and $r=2(p-1)$. For a discussion of the nature and significance of this class, see the introductions to [2], [4], [7].

Suppose $P$ is a $(p r-1)$-dimensional oriented Poincaré complex. Let $\nu$ be the stable normal spherical fibration of $P$, i.e., the unique stable spherical fibration with reducible Thom complex [6]. Define $e_{1}(P)$ to be $e_{1}(\nu)$. Similarly, define $q_{i}(P)$ to be $q_{i}(\nu)$, where $q_{i}$ is the $i$ th $\mathrm{Wu}$ class. It is clear that $e_{1}(P)$ depends only on the homotopy type of $P$. We wish, however, to express $e_{1}(P)$ in terms of an explicit invariant of the homotopy type of $P$.

In fact, we will construct a certain nonstable secondary cohomology operation $\Omega$, mapping cohomology classes of dimension $r$ into classes of dimension $p r-1$ ( $Z_{p}$ coefficients), such that

TheOREM 1. $\Omega\left(q_{1} P\right)$ is defined with zero indeterminacy, and $\Omega\left(q_{1} P\right)=e_{1}(P)$.

REMARK. In the course of the proof of Theorem 1, we will give a construction of the class $e_{1}$ which is rather different from that given by Gitler and Stasheff.

Theorem 1 allows us to compute $e_{1}(P)$-at least in principle, and often in practice. For example,

TheOREM 2. There is a Poincaré complex $P$ of the homotopy type of

$$
\left(S^{r} \vee S^{(p-1) r-1}\right) \cup e^{p r-1}
$$

such that $e_{1}(P) \neq 0$.

In another paper these results will be applied to the study of the existence of differential structures on manifolds of dimension $p r-1$. Indeed, let $\Gamma_{i}$ be the group of exotic $i$-dimensional spheres, and let $\Gamma_{i}^{\prime}$ be the quotient of $\Gamma_{i}$ by the subgroup of spheres bounding parallelizable manifolds. According to [3], $p$-torsion first appears in the groups $\Gamma_{i}^{\prime}$ precisely when $i=p r-2$; in fact, if ${ }^{p} G$ denotes the $p$-primary component of $G$, then ${ }^{p} \Gamma_{1 r-2}^{\prime}=Z_{p}$. Moreover, $\Gamma_{i}=\Gamma_{i}^{\prime}$ for $i$ even, so ${ }^{p} \Gamma_{p r-2}=Z_{p}$. Now let $M$ be a differential manifold (all manifolds are compact and oriented) of

Received by the editors June 2, 1969.

(1) The author was a National Science Foundation Postdoctoral Fellow and is partially supported by the Air Force Office of Scientific Research, Office of Aerospace Research, under Contract No. F44620-67-C-0029. 
dimension $p r-1$, whose boundary $\partial M$ is an exotic sphere. Let $[\partial M]_{p} \in{ }^{p} \Gamma_{p r-2}$ be the $p$-primary component of this exotic sphere. Also, let $M^{*}$ be the closed topological manifold $M \cup$ Cone $(\partial M)$.

THEOREM 3. $[\partial M]_{p}=0$ if and only if $\Omega\left(q_{1} M^{*}\right)=0$.

Thus $\Omega\left(q_{1} M^{*}\right)$ measures the $p$-primary component of the obstruction to smoothing $M^{*}$. Using this result, one can construct a manifold $M$ whose boundary is a generator $\Sigma_{p}$ of ${ }^{p} \Gamma_{p r-2}$.

THEOREM 4. $\Sigma_{p}$ bounds a manifold of the homotopy type of $S^{r} \vee S^{(p-1) r-1}$.

We postpone proofs of Theorems 3 and 4 to a later paper, where results on higher-dimensional exotic spheres not bounding parallelizable manifolds will also be given.

REMARK. A proof of Theorem 4 for the case $p=3$ was given in [1]. A similar proof does not work when $p \geqq 5$ because the appropriate element of $\pi_{p r-2}\left(S^{r}\right)$ is in the image of the $J$-homomorphism only when $p=3$. In particular, $\Sigma_{p}$ bounds a "plumbed" manifold only when $p=3$.

The proof of Theorem 1 uses recent techniques of $E$. Thomas. I am very grateful to Professor Thomas for teaching me about his methods.

1. Definition of the operation $\Omega$. Let $q_{i} \in H^{i r}\left(B S O ; Z_{p}\right)$ be the $i$ th $\mathrm{Wu}$ class; let $P^{i}$ be the $i$ th Steenrod power (for the prime $p$ ).

LEMMA 1.1. If $j<p$, then

$$
q_{j} \doteq b_{j} P^{j-1}\left(q_{1}\right)+R_{j}\left(q_{1}, P^{1}\left(q_{1}\right), \ldots, P^{j-2}\left(q_{1}\right)\right),
$$

where $b_{j} \in Z_{p}$ is nonzero, and $R_{j}$ is a polynomial in $j-1$ variables.

Proof. If $j=1$, clear. Assume the lemma for all $t<j$; let $U$ be the universal Thom class. Then

$$
\begin{aligned}
q_{j} \cdot U=P^{j}(U) & =b_{j} P^{j-1} P^{1}(U), \text { by an Adem relation } \\
& =b_{j} P^{j-1}\left(q_{1} \cdot U\right) \\
& =b_{j}\left[P^{j-1}\left(q_{1}\right) \cdot U+\sum_{t} P^{j-1-t}\left(q_{1}\right) \cdot P^{t}(U)\right],
\end{aligned}
$$

where the summation runs from 1 to $j-1$,

Therefore,

$$
=b_{j}\left[P^{j-1}\left(q_{1}\right) \cdot U+\sum_{t} P^{j-1-t}\left(q_{1}\right) \cdot q_{t} \cdot U\right] \text {. }
$$

$$
q_{j}=b_{j}\left[P^{j-1}\left(q_{1}\right)+\sum_{t} P^{j-1-t}\left(q_{1}\right) \cdot q_{t}\right] .
$$

Since, by assumption, $q_{t}$ is a polynomial in $q_{1}, \ldots, P^{t-1}\left(q_{1}\right)$, the lemma follows by induction. 
To define the operation $\Omega$, we will display its universal example. Let $K_{i}(i>0)$ be an Eilenberg-MacLane space $K\left(Z_{p}, i\right)$ and let $u_{i}$ be the fundamental class in $H^{i}\left(K_{i}\right)\left(Z_{p}\right.$ coefficients always).

We define a class $v \in H^{(p-1) r}\left(K_{r}\right)$ by the formula

$$
v=b_{p-1} P^{p-2}\left(u_{r}\right)+R_{p-1}\left(u_{r}, P^{1}\left(u_{r}\right), \ldots, P^{p-3}\left(u_{r}\right)\right),
$$

where $b_{p-1}$ and $R_{p-1}$ are as in Lemma 1.1 .

We have the diagram

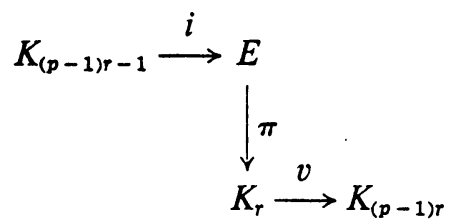

where $\pi$ is the fibration induced by $v$ from the path-loop fibration on $K_{(p-1) r}$, and $i$ is the inclusion of the fiber. (We refer to such a diagram as the principal fibration determined by $v$.)

Associated to this fibration we have the Thomas exact sequence ([10, p. 187]; see also, [5], [11])

$$
H^{j}\left(K_{r}\right) \stackrel{\pi}{\longrightarrow} H^{j}(E) \stackrel{\mu}{\longrightarrow} H^{j}\left(K_{(p-1) r-1} \times E, E\right) \stackrel{\tau}{\longrightarrow} H^{j+1}\left(K_{r}\right),
$$

valid for all $j<2(p-1) r-2$.

Define $\alpha \in H^{p r-1}\left(K_{(p-1) r-1} \times E, E\right)$ by

$$
\alpha=P^{1} u \otimes 1+u \otimes \pi^{*}\left(u_{r}\right)
$$

where $u=u_{(p-1) r-1}$.

LEMMA 1.2. $\tau(\alpha)=0$.

Proof. Using properties of $\tau[10$, p. 188], we have

$$
\tau(\alpha)=P^{1}(\bar{\tau}(u))+\bar{\tau}(u) \cup u_{r}=P^{1} v+v \cup u_{r},
$$

where $\bar{\tau}$ is the usual transgression.

Let $f: B S O \rightarrow K_{r}$ satisfy $f^{*}\left(u_{r}\right)=q_{1}$. By [2, Lemma 3.3], kernel $f^{*}$ is generated, in dimensions $\leqq p r$, by those elements in the Cartan basis containing a Bockstein. Therefore, it suffices to show $f^{*}(\tau(\alpha))=0$. But note that $f^{*}(v)=q_{p-1}$. Thus

which proves the lemma.

$$
\begin{aligned}
f^{*}(\tau(\alpha))=f^{*}\left(P^{1} v+v \cup u_{r}\right) & =P^{1}\left(q_{p-1}\right)+q_{p-1} \cup q_{1} \\
& =0 \quad \text { (by a Wu formula) }
\end{aligned}
$$

Since $\tau(\alpha)=0$, there is, by exactness of $\left(^{*}\right)$, an $\omega \in H^{p r-1}(E)$ with $\mu(\omega)=\alpha$. We take $\omega$ to be the universal representative for the operation $\Omega$. Thus $\Omega$ is defined on those classes $x \in H^{r}(X)$ for which $v(x)=0$; the indeterminacy subgroup of $\Omega(x)$ is the image of the homomorphism $H^{(p-1) r-1}(X) \rightarrow H^{p r-1}(X)$ given by (see [10], [5]) $c \rightarrow P^{1} c+c \cup x$. 
2. A result of Thomas. We quote a result which we will need in the proof of Theorem 1. Suppose we have a principal fibration

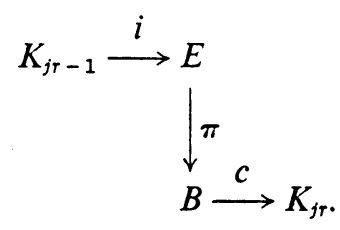

Let $k \in H^{t}(E)$, where $t<2 j r-2$, and let $i^{*}(k)=\alpha\left(u_{j r-1}\right)$ for some $\alpha$ in $A_{p}$, the Steenrod algebra. Assume there is a spherical fibration $\xi$ over $B$ with $q_{j}(\xi)=c$. Moreover, assume there is an Adem relation $\alpha P^{j}=0$, and let $\Phi$ be an associated secondary operation. Let $U_{E}$ be the Thom class of $\pi^{*}(\xi)$. Note that $\Phi$ is defined on $U_{E}$.

Proposition 2.1. There is a class $d \in H^{t}(E)$ such that $i^{*}(d)=i^{*}(k)$ and

$$
d \cdot U_{E} \in \Phi\left(U_{E}\right) \text {. }
$$

Proof. This is a special case of the $\bmod p$ analogue of Theorem 6.4 of [9]. (Set $B^{\prime}=$ point, $k^{\prime}+p^{*} m=d$ in that theorem. Thomas states his result only for vector bundles, but the proof is the same when $\xi$ is a spherical fibration.)

3. Proof of Theorem 1. We deduce Theorem 1 from a more general result. Let $\beta$ be a $(k-1)$-dimensional oriented spherical fibration over a complex $X$. Let $T(\beta)$ be the Thom complex of $\beta$ and $U_{\beta}$ the Thom class in $H^{k}(T(\beta))$. Let $\Phi$ be the secondary cohomology operation associated to the Adem relation $P^{1} P^{p-1}=0$. Assume that the Wu class $q_{p-1}(\beta)=0$. Note that $\Phi\left(\dot{U}_{\beta}\right)$ is then defined.

TheOREM A. Given $\beta$ as above, $\Omega\left(q_{1}(\beta)\right)$ is defined and

$$
\left[\Omega\left(q_{1}(\beta)\right)-e_{1}(\beta)\right] \cdot U_{\beta}=\Phi\left(U_{\beta}\right) .
$$

In particular, these two expressions have the same indeterminacy.

Before proving Theorem A, we give the

Proof of Theorem 1. Let $v$ be the normal spherical fibration of $P$. Since $T(v)$ is $S$-dual to $P^{+}$(=P with a disjoint basepoint), $P^{p-1}\left(U_{v}\right)=0$ if and only if the homomorphism

$$
c\left(P^{p-1}\right): H^{r-1}(P) \rightarrow H^{p r-1}(P)
$$

is trivial, where $c$ is the canonical anti-isomorphism of $A_{p}$. (Compare [8, Chapter III, Proposition 1.4].) But $c\left(P^{p-1}\right)$ is a multiple of $P^{p-1}$, and $P^{p-1}$ is zero on $(r-1)$-dimensional classes. Therefore $P^{p-1}\left(U_{v}\right)=0$, i.e., $q_{p-1}(\nu)=0$.

Since $q_{p-1}(\nu)=0$, we may apply Theorem A to $\nu$. Now $T(\nu)$ is reducible (that is, the top cohomology class is spherical), so $\Phi\left(U_{v}\right)=0$, with zero indeterminacy. Therefore

$$
\left[\Omega\left(q_{1}(v)\right)-e_{1}(v)\right] \cdot U_{v}=0,
$$

so $\Omega\left(q_{1} P\right)=e_{1}(P)$, with zero indeterminacy, which proves Theorem 1 . 
Proof of Theorem A. First note that $v\left(q_{1}(\beta)\right)=q_{p-1}(\beta)=0$, so, $\Omega$ is defined on $q_{1}(\beta)$.

Also, the indeterminacy of $\Phi\left(U_{\beta}\right)$ consists of all elements of the form $P^{1}(c \cdot U)$, $c \in H^{(p-1) r-1}(X)$. If $\left\{P^{1}(c \cdot U)\right\}$ denotes the set of all such elements, then

$$
\begin{aligned}
\left\{P^{1}(c \cdot U)\right\} & =\left\{\left[P^{1}(c)+c \cup q_{1}(\beta)\right] \cdot U\right\} \\
& =\left[\text { indeterminacy of } \Omega\left(q_{1}(\beta)\right)\right] \cdot U .
\end{aligned}
$$

Therefore $\left[\Omega\left(q_{1}(\beta)\right)-e_{1}(\beta)\right] \cdot U_{\beta}$ and $\Phi\left(U_{\beta}\right)$ have the same indeterminacy. Thus to prove Theorem $A$, we need only show they have a common representative.

Let $B F(m), m$ large, be the classifying space for $(m-1)$-dimensional oriented spherical fibrations, and let $\xi$ be the universal $(m-1)$-dimensional fibration.

Consider the principal fibration

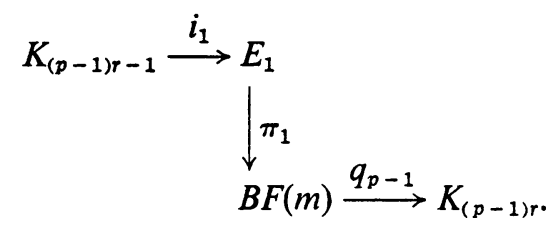

Since $q_{p-1}=v\left(q_{1}\right)$, we have a commutative diagram

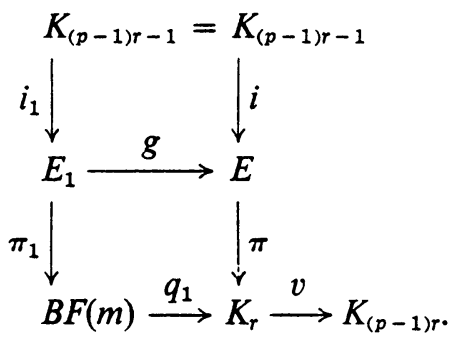

Let $k=g^{*}(\omega)$. Then, by definition, $k \in \Omega\left(\pi_{1}^{*}\left(q_{1}\right)\right)$.

Let $\delta$ over $E_{1}$ be the spherical fibration $\pi_{1}^{*}(\xi)$. We claim

$$
\left(k-e_{1}(\delta)\right) \cdot U_{\delta} \in \Phi\left(U_{\delta}\right) .
$$

Notice that Theorem A follows immediately from $\left({ }^{*}\right)$, for if $\beta: X \rightarrow B F(m)$ is a spherical fibration with $q_{p-1}(\beta)=0$, then $\beta$ lifts to $f: X \rightarrow E_{1}$. By naturality, $\left(f^{*}(k)-e_{1}(\beta)\right) \cdot U_{\beta} \in \Phi\left(U_{\beta}\right)$ and $f^{*}(k) \in \Omega\left(q_{1}(\beta)\right)$. Thus $\left(f^{*}(k)-e_{1}(\beta)\right) \cdot U_{\beta}$ is the required common representative.

To show $\left(^{*}\right)$, we apply Proposition 2.1 to the fibration (3.1), with $\alpha=P^{1}$. We conclude that there is a class $d \in H^{p r-1}\left(E_{1}\right)$ with $i^{*}(d)=i^{*}(k)$ and

$$
d \cdot U_{\delta} \in \Phi\left(U_{\delta}\right) \text {. }
$$

LEMMA 3.2. $k=d+\pi_{1}^{*}(z)$, for a unique $z \in H^{p r-1}(B F(m))$.

Proof. It is easy to see that $H^{i}(B F)=0, i<r$, and $H^{r}(B F)=Z_{p}$, with $q_{1}$ as 
generator. (For example, see [4].) It follows that any $\alpha \in H^{p r-1}\left(K_{(p-1) r-1} \times E_{1}, E_{1}\right)$ can be written in the form

Let

$$
a P^{1}(u) \otimes 1+u \otimes \pi_{1}^{*}\left(b \cdot q_{1}\right), \quad a, b \in Z_{p} .
$$

$$
\mu: H^{p r-1}\left(E_{1}\right) \rightarrow H^{p r-1}\left(K_{(p-1) r-1} \times E_{1}, E_{1}\right)
$$

be the map in the Thomas exact sequence for the fibration (3.1). If $i_{1}^{*}(x)=0$, $x \in H^{p r-1}\left(E_{1}\right)$, then $\mu(x)=u \otimes \pi_{1}^{*}\left(b \cdot q_{1}\right)$. Now $0=\tau \mu(x)=b\left(q_{p-1} \cup q_{1}\right)$. This implies $b=0$, since $q_{p-1} \cup q_{1} \neq 0$. Therefore, $i_{1}^{*}(x)=0$ implies $\mu(x)=0$. By exactness, $x=\pi_{1}^{*}(z)$. Moreover, a glance at the Thomas exact sequence shows that

$$
\pi_{1}^{*}: H^{p r-1}(B F(m)) \rightarrow H^{p r-1}\left(E_{1}\right)
$$

is injective, so $z$ is unique. Since $i_{1}^{*}(k-d)=0$, the lemma follows.

LEMMA 3.3. The class $z$ which occurs in Lemma 3.2 is nonzero.

Proof. Let $h: S^{p r-1} \rightarrow B F(m)$ be a generator of ${ }^{p} \pi_{p r-1}(B F(m))=Z_{p}$. Note that $h$ lifts to $f: S^{p r-1} \rightarrow E_{1}$. By Lemma 3.2, $f^{*}(k)=f^{*}(d)+h^{*}(z)$.

Now $f^{*}(k) \in \Omega\left(q_{1}(h)\right)=0$, with zero indeterminacy, so $f^{*}(d)=-h^{*}(z)$.

By $\left({ }^{* *}\right), f^{*}(d) \cdot U_{h} \in \Phi\left(U_{h}\right)$.

But the Thom complex $T(h)$ is of the form $S^{m} \cup_{\beta_{1}} e^{m+p r-1}$, where $\beta_{1}$ is a generator of ${ }^{p} \pi_{p r-2}$ (see [2]), and $\Phi$ acts nontrivially in this complex, i.e., $\Phi\left(U_{h}\right) \neq 0$, with zero indeterminacy. Therefore $z \neq 0$.

We now define $e_{1}$ to be the class $z$. Then

$$
d=k-\pi_{1}^{*}\left(e_{1}\right)=k-e_{1}(\delta) .
$$

Together with $\left({ }^{* *}\right)$, this shows $\left({ }^{*}\right)$, and proves Theorem A. Alternatively, we may show that $z$ is equal to the class defined in [2], which we will call $\bar{e}_{1}$. Since $H^{p_{r-1}}(B F)$ is at most $Z_{p}$ [4], $\bar{e}_{1}=b z, b \in Z_{p}$ nonzero. According to [2], if $\Psi$ is the secondary operation corresponding to the relation $P^{1}\left(P^{1}\right)^{p-1}=0$, then $h^{*}\left(\bar{e}_{1}\right)$ - $U_{h} \in \Psi\left(U_{h}\right)$. Now $\left(P^{1}\right)^{p-1}=(p-1) ! P^{p-1}=-P^{p-1}$, so $-h^{*}\left(\bar{e}_{1}\right) \cdot U_{h} \in \Phi\left(U_{h}\right)$. But $-h^{*}(z) \cdot U_{h} \in \Phi\left(U_{h}\right)$. Therefore $\bar{e}_{1}=z$.

4. Proof of Theorem 2. Theorem 2 follows easily from

Proposition 4.1. There is a $\beta \in \pi_{p r-2}\left(S^{r}\right)$ such that if $X=S^{r} \cup_{\beta} e^{p r-1}$, then $\Omega: H^{r}(X) \rightarrow H^{p r-1}(X)$ is nontrivial. (Clearly $\Omega$ is defined with zero indeterminacy.)

Assuming Proposition 4.1, we prove Theorem 2. Recall that

$$
\pi_{i+j-1}\left(S^{i} \vee S^{j}\right)=\pi_{i+j-1}\left(S^{i}\right) \oplus \pi_{i+j-1}\left(S^{j}\right) \oplus Z, \quad(i, j \geqq 2)
$$

where the infinite cyclic factor is generated by the Whitehead product $\left[e_{i}, e_{j}\right]$ of the inclusions $e_{t}: S^{t} \rightarrow S^{i} \vee S^{j}, t=i, j$.

If $f \in \pi_{i+j-1}\left(S^{i} \vee S^{j}\right)$, we write

$$
f=f_{i} \oplus f_{j} \oplus H_{f}, \quad f_{t} \in \pi_{i+j-1}\left(S^{t}\right), \quad H_{f} \in Z .
$$


If $i \neq j$, it is easy to see that $\left(S^{i} \vee S^{j}\right) \cup \cup_{f} e^{i+j}$ is a Poincare complex if and only if $H_{f}= \pm 1$. We write $P\left(f_{i}, f_{j}\right)$ for the Poincaré complex $\left(S^{i} \vee S^{j}\right) \cup{ }_{f} e^{i+j}$, where $f=f_{i} \oplus f_{j} \oplus 1$. Now let $i=r, j=(p-1) r-1$. Let $\alpha \in \pi_{p r-2}\left(S^{(p-1) r-1}\right)$ be a map such that $P^{1}: H^{(p-1) r-1}(Y) \rightarrow H^{p r-1}(Y)$ is nontrivial, where $Y=S^{(p-1) r-1}$ $\cup_{\alpha} e^{p r-1}$ (see [8, p. 89]). Let $P=P(\beta, \alpha)$, with $\beta$ as in Proposition 4.1.

We claim $e_{1}(P) \neq 0$. By Theorem $1, \Omega\left(q_{1} P\right)$ is defined with zero indeterminacy, and $e_{1}(P)=\Omega\left(q_{1} P\right)$. But there is a map $c: P \rightarrow X=S^{r} \cup_{\beta} e^{p r-1}$ such that $c^{*}: H^{t}(X)$ $\rightarrow H^{t}(P)$ is an isomorphism for $t \neq(p-1) r-1$ (collapse $S^{(p-1) r-1}$ to a point). By naturality, $\Omega: H^{r}(P) \rightarrow H^{p r-1}(P)$ is an isomorphism. Thus it suffices to show $q_{1}(P) \neq 0$; this follows from the fact that $P^{1}: H^{(p-1) r-1}(P) \rightarrow H^{p r-1}(P)$ is nontrivial.

This proves Theorem 2, except for the proof of Proposition 4.1, which we now give. The following lemma is certainly known to the dedicated homotopy theorists (for completeness, we give a proof).

LEMMA 4.2. There exist a complex

$$
L=S^{2 r-2} \cup e^{3 r-2} \cup \cdots \cup e^{(p-1) r-2}
$$

and maps

$$
g: L \rightarrow S^{r-1}, \quad h: S^{p r-3} \rightarrow L
$$

such that

(1) $P^{1}: H^{i r-2}(L) \rightarrow H^{(i+1) r-2}(L)$ is nontrivial, $2 \leqq i \leqq p-2$.

(2) The functional operation $P_{g}^{1}: H^{r-1}\left(S^{r-1}\right) \rightarrow H^{2 r-2}(L)$ is nontrivial.

(3) The functional operation $P_{h}^{1}: H^{(p-1) r-2}(L) \rightarrow H^{p r-3}\left(S^{p r-3}\right)$ is nontrivial.

Proof. Suppose inductively that we have constructed a complex

$$
L(i-1)=S^{2 r-2} \cup e^{3 r-2} \cup \cdots \cup e^{(i-1) r-2}, \quad i \leqq p-1,
$$

and a map $g(i-1): L(i-1) \rightarrow S^{r-1}$ such that the functional operation $P_{g(i-1)}^{1}$ is nontrivial.

We define

$$
L(i)=\sum\left[S^{r-1} \cup_{g(i-1)} \text { Cone }(L(i-1))\right],
$$

where $\sum$ is the $(r-1)$-fold suspension.

To define $g(i): L(i) \rightarrow S^{r-1}$, we use the following lemma.

LEMMA 4.3. Let $(A, B)$ be a pair of complexes which is the suspension of a pair $\left(A^{\prime}, B^{\prime}\right)$, and let $Y$ be a space which is n-simple for all $n$. Suppose $f: B \rightarrow Y$ is of order $p$ in the group of homotopy classes $[B, Y]$. If, for all $i, H^{i+1}\left(A, B ; \pi_{i}(Y)\right)$ is finite and has trivial p-primary component, then $f$ extends over $A$.

Proof. Left to reader. (Hint: Given a partial extension $F$ of $f$, the obstruction cohomology class $c_{F}$ has order $t, t \not \equiv 0(p)$. Take $s$ with $s t \equiv 1(p)$. Then $s t(F) \mid B=f$ and $c_{s t(F)}=0$.) 
Let $\alpha: S^{2 r-2} \rightarrow S^{r-1}$ be a map which is of order $p$ in $\pi_{2 r-2}\left(S^{r-1}\right)$; then the functional operation $P_{\alpha}^{1}$ is nontrivial [8, p. 90]. Since ${ }^{p} \pi_{j r-3}\left(S^{r-1}\right)=0$ for $j \leqq p-1$ [12], Lemma 4.3 implies that $\alpha$ extends to a map $g(i): L(i) \rightarrow S^{r-1}$.

Thus we may construct $L(p-1)$ and $g(p-1): L(p-1) \rightarrow S^{r-1}$. We may also construct the complex $L(p)$ (but not the map $g(p)$ ). Let

$$
\begin{aligned}
L & =((p-1) r-2) \text {-skeleton of } L(p), \\
h: S^{p r-3} \rightarrow L & =\text { attaching map of the }(p r-2) \text {-cell of } L(p), \\
g: L \rightarrow S^{r-1} & =\text { an extension of } \alpha: S^{2 r-2} \rightarrow S^{r-1} .
\end{aligned}
$$

It is easy to see that the $L, g$, and $h$ we have constructed satisfy conditions (1), (2), and (3).

Now let $\beta^{\prime} \in \pi_{p r-3}\left(S^{r-1}\right)$ be the composite $g h$, and let $\beta$ be the suspension of $\beta^{\prime}$. Also, let

$$
X^{\prime}=S^{r-1} \cup_{\beta^{\prime}} e^{p r-2}, \quad X=\text { suspension of } X^{\prime}=S^{r} \cup_{\beta} e^{p r-1} .
$$

To show $\Omega: H^{r}(X) \rightarrow H^{p r-1}(X)$ is nontrivial, it suffices to show

$$
\Omega^{\prime}: H^{r-1}\left(X^{\prime}\right) \rightarrow H^{p r-2}\left(X^{\prime}\right)
$$

is nontrivial, where $\Omega^{\prime}$ is the secondary operation whose universal example is obtained by applying the loop functor to the universal example for $\Omega$. Thus the universal example for $\Omega^{\prime}$ is

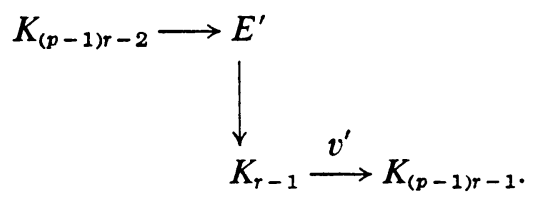

Since $v=b P^{p-2}+$ product terms, $b \in Z_{p}$ nonzero, $v^{\prime}=b P^{p-2}$. (Informally, $\Omega^{\prime}$ is the secondary operation associated to the relation $P^{1} P^{p-2}=0$ on classes of dimension $r-1$.)

There is a commutative ladder of cofibrations

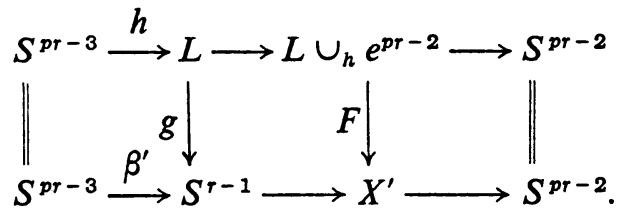

Using conditions (1) and (2) of Lemma 4.2 (and the fact that $P^{p-2}=b^{\prime}\left(P^{1}\right)^{p-2}$ ), we see that the functional operation

$$
P_{F}^{p-2}: H^{r-1}\left(X^{\prime}\right) \rightarrow H^{(p-1) r-2}\left(L \cup e^{p r-2}\right)
$$

is nontrivial. Using condition (3) of Lemma 4.2, we see that

$$
P^{1}: H^{(p-1) r-2}\left(L \cup_{h} e^{p r-2}\right) \rightarrow H^{p r-2}\left(L \cup_{h} e^{p r-2}\right)
$$


is nontrivial. A Peterson-Stein formula [5] shows that

$$
\Omega^{\prime}: H^{r-1}\left(X^{\prime}\right) \rightarrow H^{p r-2}\left(X^{\prime}\right)
$$

is nontrivial. This completes the proof of Proposition 4.1.

\section{REFERENCES}

1. D. Frank, An invariant for almost-closed manifolds, Bull. Amer. Math. Soc. 74 (1968), 562-567.

2. S. Gitler and J. Stasheff, The first exotic class of BF, Topology 4 (1965), 257-266.

3. M. Kervaire and J. Milnor, Groups of homotopy spheres, Ann. of Math. 77 (1963), 504-537.

4. J. Milnor, On characteristic classes for spherical fiber spaces, Comment. Math. Helv. 43 (1968), 51-77.

5. F. Peterson and N. Stein, Secondary cohomology operations: two formulas, Amer. J. Math. 81 (1959), 281-305.

6. M. Spivak, Spaces satisfying Poincaré duality, Topology 6 (1967), 77-102.

7. J. Stasheff, More characteristic classes for spherical fiber spaces, Comment. Math. Helv. 43 (1968), 78-86.

8. N. Steenrod and D. Epstein, Cohomology operations, Ann. of Math. Studies No. 50, Princeton Univ. Press, Princeton, N. J., 1962.

9. E. Thomas, The index of a tangent 2-field, Comment. Math. Helv. 42 (1967), 86-110.

10. - Postnikov invariants and higher order cohomology operations, Ann. of Math. 85 (1967), 184-217.

11. - Seminar on fiber spaces, Springer-Verlag, Berlin, 1966.

12. H. Toda, Composition methods in homotopy groups of spheres, Ann. of Math. Studies No. 49, Princeton Univ. Press, Princeton, N. J., 1962.

Massachusetts Institute of Technology, Cambridge, Massachusetts 